\title{
Entomofauna aérea em Eucalyptus spp. no Sudeste do Pará, Brasil
}

\author{
Aerial entomofauna in Eucalyptus spp. in Southeast Pará, Brazil \\ Entomofauna aérea en Eucalyptus spp. en el Sureste de Pará, Brasil
}

Recebido: 30/10/2021 | Revisado: 09/11/2021 | Aceito: 10/11/2021 | Publicado: 15/11/2021

Ivy Laura Saliba

ORCID: https://orcid.org/0000-0001-6732-4112

Universidade Federal Rural da Amazônia, Brasil

E-mail: ivysaliba@hotmail.com

Alexandre Mehl Lunz

ORCID: https://orcid.org/0000-0002-4107-0879

Embrapa Amazônia Oriental, Brasil

E-mail: alexandre.mehl@embrapa.br

Telma Fátima Vieira Batista

ORCID: https://orcid.org/0000-0001-7379-6028

Universidade Federal Rural da Amazônia, Brasil

E-mail: telma.batista@ufra.edu.br

Luiz Fernandes Silva Dionisio

ORCID: https://orcid.org/0000-0002-4324-2742

Universidade do Estado do Pará, Brasil

E-mail: fernandesluiz03@gmail.com

Helton Bastos Machado

ORCID: https://orcid.org/0000-0002-1948-3108

Universidade Federal Rural da Amazônia, Brasil

E-mail: helton-machado@hotmail.com

Gustavo Schwartz

ORCID: https://orcid.org/0000-0002-1717-4491

Embrapa Amazônia Oriental, Brasil

E-mail: gustavo.schwartz@embrapa.br

\begin{abstract}
Resumo
Plantios florestais homogêneos são comumente atacados por agentes bióticos devido à menor diversidade biológica. Este tipo de plantio, especialmente de eucalipto, se intensificou no sudeste do estado do Pará, Brasil, a partir dos anos 1990. Desta forma, o objetivo deste estudo foi prospectar a diversidade da entomofauna aérea, avaliar suas respostas à sazonalidade e detectar a ocorrência de possíveis insetos praga importantes para monocultivos de Eucalyptus spp. Foram utilizadas armadilhas amarelas adesivas para a amostragem em plantios de E. urophylla e do híbrido $E$. grandis x E. urophylla. No total, foram realizadas 12 coletas, com 27 armadilhas cada, divididas em estação seca e chuvosa, durante dois anos em três municípios do Sudeste do Pará. Foram calculados os índices de diversidade de ShannonWiener e Pielou. As famílias foram classificadas quanto à diversidade faunística, através dos índices de dominância, frequência, abundância e constância. $\mathrm{O}$ teste de análise Fatorial $2 \times 2$ foi realizado para comparar o efeito da sazonalidade entre os eucaliptos. Foram coletados 10.280 indivíduos, distribuídos em oito ordens e 68 famílias. As ordens mais abundantes foram Hemiptera, Coleoptera e Diptera, onde Hemiptera foi a mais abundante na estação seca, e Diptera na estação chuvosa. As famílias Aphalaridae e Thaumastocoridae foram as mais importantes. Também foi registrada a ocorrência das espécies praga Blastopsylla occidentalis, Glycaspis brimblecombei e Thaumastocoris peregrinus.
\end{abstract}

Palavras-chave: Insetos praga; Sazonalidade; Armadilha adesiva; Aphalaridae; Thaumastocoridae.

\begin{abstract}
Homogeneous forest plantations are commonly attacked by biotic agents due to their lower biological diversity. This type of planting, especially of eucalyptus, was intensified in Southeast of the state of Pará, Brazil, from the 1990s onwards. Thus, the objective of this study was to prospect the diversity of aerial entomofauna, evaluate their responses to seasonality, and detect the occurrence of possible important pest insects for monocultures of Eucalyptus spp. Yellow adhesive traps were used for sampling in plantations of E. urophylla and the hybrid E. grandis $\mathrm{x} E$. urophylla. In total, 12 collections were carried out, with 27 traps each, divided into dry and rainy seasons, during two years in three municipalities in the Southeast of Pará. The Shannon-Wiener and Pielou diversity indices were calculated. Families were classified according to their faunal diversity, through the dominance, frequency, abundance, and constancy indices. The $2 \times 2$ factorial analysis test was performed to compare the effect of seasonality between the eucalyptus. A total of 10,280 individuals were collected, distributed in eight orders and 68 families. The most abundant orders were Hemiptera, Coleoptera, and Diptera, where Hemiptera was the most abundant in the dry season,
\end{abstract}


and Diptera in the rainy season. The Aphalaridae and Thaumastocoridae families were the most important. The occurrence of the pest species Blastopsylla occidentalis, Glycaspis brimblecombei, and Thaumastocoris peregrinus was also recorded.

Keywords: Pest insects; Seasonality; Adhesive trap; Aphalaridae; Thaumastocoridae.

\section{Resumen}

Plantaciones forestales homogéneas son atacadas habitualmente por agentes bióticos debido a su menor diversidad biológica. Este tipo de siembra, especialmente de eucalipto, se intensificó en el sureste del estado de Pará, Brasil, a partir de la década de 1990. Así, el objetivo de este estudio fue prospectar la diversidad de entomofauna aérea, evaluar sus respuestas a la estacionalidad y detectar la ocurrencia de posibles insectos plaga importante para monocultivos de Eucalyptus spp. Se utilizaron trampas adhesivas amarillas para el muestreo en plantaciones de E. urophylla y el híbrido E. grandis x E. urophylla. En total, se realizaron 12 colectas, con 27 trampas cada una, divididas en temporadas seca y lluviosa, durante dos años en tres municipios del sureste de Pará. Se calcularon los índices de diversidad de Shannon-Wiener y Pielou. Las familias se clasificaron según su diversidad de fauna, mediante los índices de dominancia, frecuencia, abundancia y constancia. Se realizó la prueba de análisis factorial 2 x 2 para comparar el efecto de la estacionalidad entre los eucaliptos. Se recolectaron 10.280 individuos, distribuidos en ocho órdenes y 68 familias. Los órdenes más abundantes fueron Hemiptera, Coleoptera y Diptera, donde Hemiptera fue el más abundante en la época seca y Diptera en la época de lluvias. Las familias Aphalaridae y Thaumastocoridae fueron las más importantes. También se registró la presencia de las especies plaga Blastopsylla occidentalis, Glycaspis brimblecombei y Thaumastocoris peregrinus.

Palabras clave: Insectos plaga; Estacionalidad; Trampa adhesiva; Aphalaridae; Thaumastocoridae.

\section{Introdução}

O eucalipto (Eucalyptus spp.: Myrtaceae) foi introduzido no Brasil em 1863 (Foelkel, 2005) para atender às indústrias siderúrgicas e o setor de celulose e papel (Costa et al, 2014). Os primeiros plantios no Pará foram iniciados em 1990 (Machado \& Maia, 2017) para atender à demanda de madeira no setor energético. A partir de 2010, o monocultivo de espécies exóticas, incluindo o eucalipto, se intensificou no Pará, especialmente no sudeste do estado, devido à substituição de atividades agropecuárias por plantios de reflorestamento. O estado do Pará possui 154.402 ha de eucalipto, sendo o décimo estado com maior plantio no país (IBA, 2020) e com potencial de expansão devido à disponibilidade de áreas para o setor florestal.

Plantios florestais homogêneos são comumente atacados por agentes bióticos considerados pragas devido à menor diversidade biológica nas áreas, quando comparadas a florestas nativas (Costa et al., 2014; Lunz et al., 2011). Pragas fitófagas como Thaumastocoris peregrinus Carpintero e Dellapé, 2006 (Hemiptera: Thaumastocoridae), Glycaspis brimblecombei (Moore, 1964) e Blastopsylla occidentalis (Taylor, 1985) (Hemiptera: Aphalaridae) podem ocorrer em plantações de eucalipto (Carpintero \& Dellapé, 2006; Saliba et al., 2019a; Saliba et al., 2019b). As árvores quando atacadas por T. peregrinus apresentam sintomas como o ressecamento e mudança de cor das folhas, de prateada natural a marrom-avermelhada e quando atacadas por G. brimblecombei e B. occidentalis também apresentam folhas ressecadas e reduzidas em número, com deformação da lâmina foliar e indução ao molde fuliginoso (Saliba et al., 2019a).

O uso de armadilhas de captura para levantamentos entomofaunísticos proporciona facilidade na coleta dos insetos, amostragem da densidade populacional e a caracterização da comunidade, sendo importante método de monitoramento e observação da maioria dos insetos-praga (Garlet et al., 2016). O estudo da entomofauna nos monocultivos de Eucalyptus spp. auxilia na detecção de pragas e no conhecimento da sazonalidade, fornecendo parâmetros para recomendações seguras e efetivas nos métodos de controle (Lima, 1996).

Entre as armadilhas para amostragem da entomofauna aérea, a utilização de armadilhas amarelas adesivas proporciona análise qualitativa e quantitativa de insetos por meio das variações na distribuição e dinâmica de suas populações em plantios de Eucalyptus spp. Com estas armadilhas nos plantios é possível observar a diversidade de famílias entomológicas, bem como suas interações ecológicas, permitindo a classificação de insetos não-praga, agentes biológicos e insetos-praga (Gallo et al., 2002; Gullan \& Cranston, 2012; Costa et al., 2014). 
Neste trabalho foi estudada a entomofauna aérea associada a plantios comerciais de Eucalyptus spp. com o objetivo de: a) Prospectar a diversidade aérea; b) Conhecer a influência da sazonalidade sobre a comunidade e c) Detectar a possível ocorrência de pragas importantes como os psilídeos G. brimblecombei e B. occidentalis e o percevejo-bronzeado T. peregrinus.

\section{Metodologia}

\section{Área de estudo}

$\mathrm{O}$ estudo foi conduzido em áreas de plantios comerciais de Eucalyptus urophylla e E. grandis x E. urophylla (clone híbrido), com idades entre 1 e 6 anos, localizados na Amazônia Oriental, sudeste do estado do Pará, Brasil. Foram selecionadas

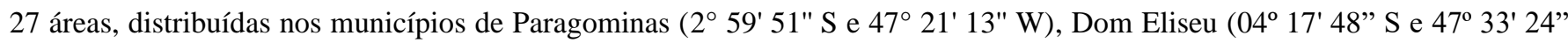
W) e Ulianópolis ( $\left(03^{\circ} 45^{\prime} 32^{\prime \prime} \mathrm{S}\right.$ e $\left.47^{\circ} 29^{\prime} 26^{\prime \prime} \mathrm{W}\right)$, onde cada área tinha cerca de 500 ha (Figura 1). As áreas foram selecionadas de acordo com: a) Maior representatividade de eucalipto em relação ao total de área dos municípios; b) Disposição das armadilhas; c) Plantios com idade entre um e seis anos e d) Proximidade a estradas vicinais.

Figura 1. Localização das áreas de amostragem da entomofauna aérea em plantios comerciais de Eucalyptus spp. nos municípios de Paragominas, Dom Eliseu e Ulianópolis, estado do Pará, Amazônia Oriental, Brasil.

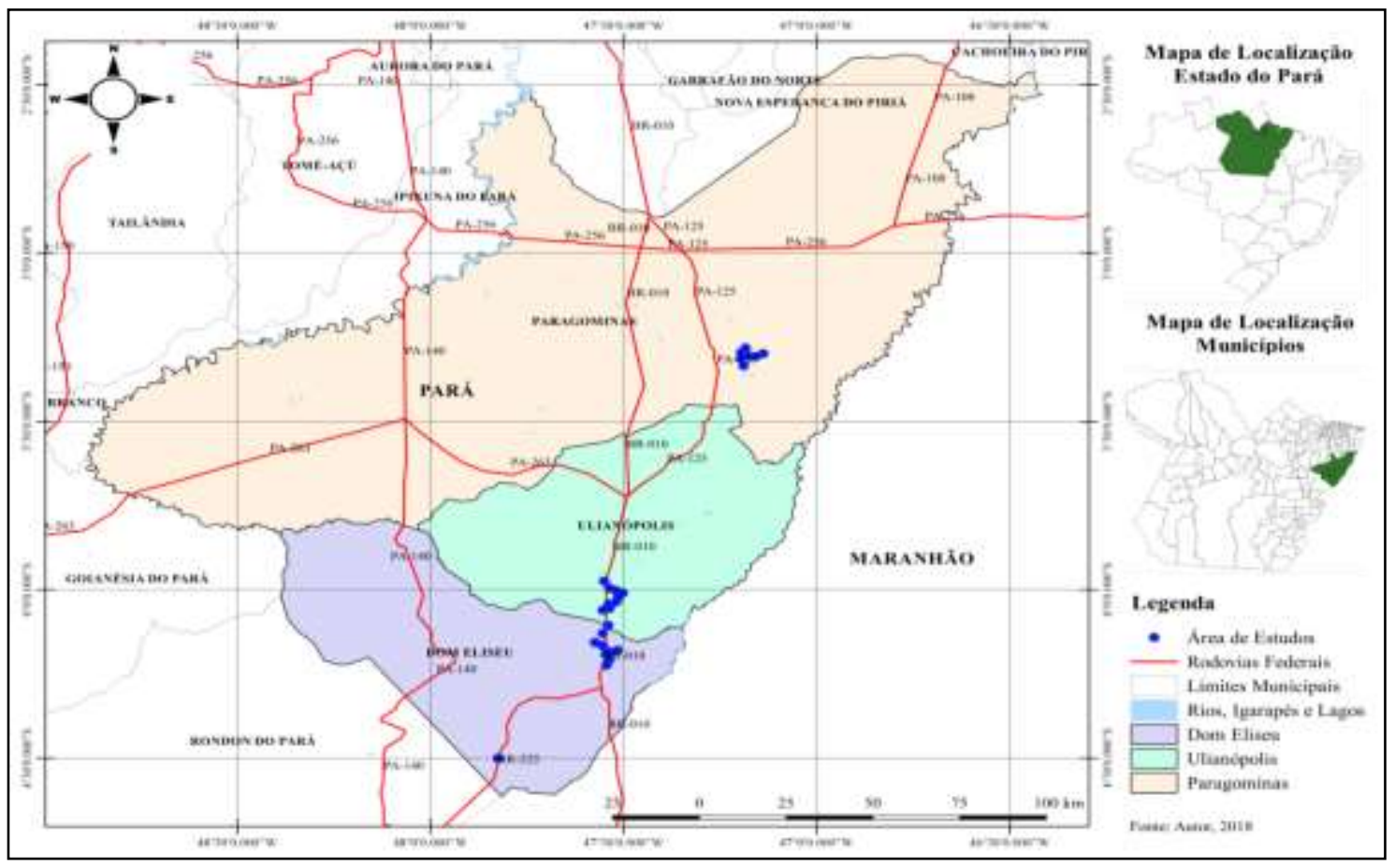

Fonte: Saliba (2019a).

A vegetação predominante original e remanescente ao redor dos plantios inclui Floresta Ombrófila Densa de Terra Firme e Floresta Densa de Planície Aluvial (IBGE, 2012; FAPESPA, 2016). Os solos predominantes na região se distribuem em Latossolo Vermelho Amarelo, textura argilosa, com relevo plano a suavemente ondulado, Latossolo Amarelo distrófico e Plintossolo pétrico, com predomínio do relevo acidentado (EMBRAPA, 2013; FAPESPA, 2016). A classificação climática, segundo Köppen, é do tipo Awi, tropical chuvoso com estação chuvosa entre janeiro e maio e estação seca bem definida entre julho e novembro e pluviosidade de $2.250 \mathrm{~mm}$ a $2.500 \mathrm{~mm}$, com média anual de $1.802 \mathrm{~mm}$. As temperaturas médias do ar variam de $25{ }^{\circ} \mathrm{C} \mathrm{a} 28{ }^{\circ} \mathrm{C}$, com umidade relativa do ar de 85\% (SIPAM, 2009; FAPESPA, 2016). 


\section{Amostragem e identificação dos insetos}

Neste estudo, o tipo de pesquisa abordado foi a pesquisa de campo quantitativa-descritiva (Gómez, 2000). O trabalho de pesquisa incluiu atividade de campo para a coleta dos insetos na área descrita acima, seguida de atividade de laboratório para a identificação dos insetos coletados. Os métodos para as coletas de insetos em campo e sua identificação em laboratório são descritos abaixo.

O método utilizado para captura dos insetos aéreos foi o uso de armadilhas adesivas amarelas $(23 \mathrm{x} 11 \mathrm{~cm})$ da marca ISCA, tendo ambos os lados quadriculados $(2,0 \times 2,0 \mathrm{~cm})$. As armadilhas foram georreferenciadas e posicionadas entre duas árvores de Eucalyptus spp., a 1,60 m do solo, com distância de $100 \mathrm{~m}$ da borda do plantio. Este método de amostragem é também utilizado para detecção de psilídeos, percevejo-bronzeado e da vespa-da-galha (Barbosa et al., 2012).

Foram realizadas três coletas em cada estação, durante dois anos consecutivos. Foram instaladas sete armadilhas no município de Paragominas, nove em Ulianópolis e $11 \mathrm{em}$ Dom Eliseu. A triagem das armadilhas foi realizada nos laboratórios de Entomologia da Embrapa Amazônia Oriental e da Universidade Federal Rural da Amazônia, em Belém, Pará. A identificação taxonômica dos insetos, na maioria, foi realizada até família com auxílio de microscópio estereoscópico e chaves taxonômicas. A espécie T. peregrinus foi identificada de acordo com Carpintero e Dellapé (2006) e Wilcken et al. (2010). Os psilídeos foram identificados pelo Dr. Daniel Burckhardt, do Museu de História Natural da Suíça, de acordo com a classificação e nomenclatura de Psylloidea descrita por Burckhardt e Ouvrard (2012) e Burckhardt e Queiroz (2012).

\section{Análise de dados}

Os insetos coletados foram analisados em função dos índices faunísticos: dominância, frequência, abundância, constância e diversidade. O Software ANAFAU, desenvolvido pelo Departamento de Entomologia da ESALQ/USP, foi utilizado para as análises.

Quanto à dominância (D), obtém-se através do total do número de indivíduos coletados por espécie em cada ano do levantamento. As espécies foram classificadas em: Não Dominantes (ND), Dominantes (D) e Superdominantes (SD), sendo consideradas não dominantes quando a frequência for menor que $1 / \mathrm{S}$ e dominantes quando a frequência for superior a $1 / \mathrm{S}$, onde $\mathrm{S}$ correspondeu à riqueza da espécie na área amostrada (Silveira Neto et al., 1976).

A frequência $(F)$ foi obtida através da soma dos dados das coletas mensais, calculando-se a percentagem de indivíduos de cada espécie em relação ao total de indivíduos coletados. A frequência calculada seguiu a fórmula descrita por Silveira Neto et al. (1976), $\mathrm{F}=\mathrm{N} / \mathrm{T}$ x 100, onde: $\mathrm{F}=$ Frequência; $\mathrm{N}$ = Total de indivíduos de cada espécie capturada; $\mathrm{T}$ = Total de indivíduos capturados. O intervalo de confiança (IC) da média com 5\% de probabilidade (Fazolin, 1991) classificou as espécies em Muito Frequentes (mf) - número de indivíduos maior que o limite superior do IC a 5\%; Frequentes (f) - número de indivíduos situados dentro do IC a 5\%; e Pouco Frequentes (pf) - número de indivíduos menor que o limite inferior do IC a 5\%.

A abundância (A) foi determinada utilizando-se as médias de dispersão, através do desvio padrão da média e intervalo de confiança (IC) (Silveira Neto et al., 1976), empregando-se o teste "t" a 5\% e 1\% de probabilidade (Dajoz, 1983), onde foram estabelecidas as seguintes classes de abundância: Rara (r): número de indivíduos menor que o limite inferior do IC a $1 \%$ de probabilidade; Dispersa (d): número de indivíduos situado entre os limites inferiores do IC a 5\% e 1\% de probabilidade; Comum (c): número de indivíduos situado dentro do IC a 5\% de probabilidade; Abundante (a): número de indivíduos situado entre os limites superiores do IC a 5\% e 1\% de probabilidade; e Muito abundante (ma): número de indivíduos maior que o limite superior do IC a $5 \%$ de probabilidade.

A Constância (C) foi calculada de acordo com a percentagem de ocorrência das espécies presentes (Silveira Neto et al., 1976), com $\mathrm{C}=\mathrm{p} / \mathrm{N}$ x 100, onde: $\mathrm{C}=$ constância; $\mathrm{p}=$ número de coletas contendo a espécie; e $\mathrm{N}$ = número total de coletas efetuadas. Teve-se as seguintes classes adotadas, segundo Dajoz (1983): Espécies constantes (W) - presentes em mais de 50\% 
das coletas; Espécies acessórias (Y) - presentes em 25 a 50\% das coletas; e Espécies acidentais (Z) - presentes em menos de $25 \%$ das coletas.

O uso do Índice de Diversidade de Shannon $\left(\mathrm{H}^{\prime}\right)$ foi usado para estimar a diversidade específica expressando a heterogeneidade de insetos nos plantios (Magurran, 2011). Quanto maior o valor de H', maior a diversidade da comunidade estudada. O índice é calculado pelas seguintes equações:

$$
H^{\prime}=-\sum p i \cdot \log p i
$$

onde: $\mathrm{H}^{\prime}=$ Índice de Diversidade de Shannon, $p i=\frac{n i}{N} ; \mathrm{ni}=$ número de indivíduos amostrados da i-ésima espécie. $\mathrm{N}=$ número total de indivíduos amostrados.

A diversidade máxima (H'max) se $\mathrm{S}=\mathrm{N}$ ou se a razão N/S for aproximadamente constante, é determinada por:

$$
H_{\text {max }}^{\prime}=\ln (s)
$$

onde, $\mathrm{S}=$ número total de espécies amostradas; $\ln$ = logaritmo neperiano.

O Índice de equabilidade de Pielou (J) foi derivado do índice de diversidade de Shannon e representa a uniformidade da distribuição dos indivíduos entre as espécies existentes. Foi considerado amplitude 0 , a uniformidade foi mínima e igual a 1 , uniformidade máxima, onde, quanto maior o valor de $\mathrm{J}$, maior foi a uniformidade da comunidade estudada, podendo ser obtido pela equação:

$$
J^{\prime}=H^{\prime} / H^{\prime} \max
$$

onde, H' = índice de diversidade de Shannon-Wiener; H'max = índice de diversidade máxima (Pielou, 1966).

Foi realizada a análise em Fatorial 2 x 2, em delineamento inteiramente casualizado, sendo os fatores os dois períodos climáticos (estação seca e chuvosa) e os clones de E. urophylla e o híbrido E. grandis x E. urophylla, com três repetições (coletas) para cada variável resposta. Os dados foram submetidos à análise de variância pelo teste F, e quando significativos, as médias foram comparadas em nível de 5\% de probabilidade, pelo mesmo teste. Para realização das análises foi utilizado o programa estatístico R versão 3.3.1. (R Core Team, 2021).

\section{Resultados}

Foram coletados 10.280 insetos distribuídos em oito ordens (Blattodea, Coleoptera, Diptera, Hemiptera, Hymenoptera, Isoptera, Lepidoptera e Orthoptera) e 68 famílias. As ordens com o maior número de indivíduos foram: Hemiptera (48\%), Diptera (21\%) e Coleoptera (21\%) nos clones de E. urophylla e no híbrido E. urophylla x E. grandis (Figura 2). Ao avaliar individualmente os diferentes clones E. urophylla e o híbrido, foi possível observar que E. urophylla apresentou um total de 4.610 indivíduos coletados, distribuídos em oito ordens e 60 famílias, enquanto o híbrido apresentou um total de 5.670 indivíduos coletados, distribuídos em oito ordens e 66 famílias. 
Figura 2. Número de indivíduos e percentagem das ordens coletadas na estação seca e chuvosa, em áreas comerciais de Eucalyptus spp. Amazônia Oriental, Brasil.

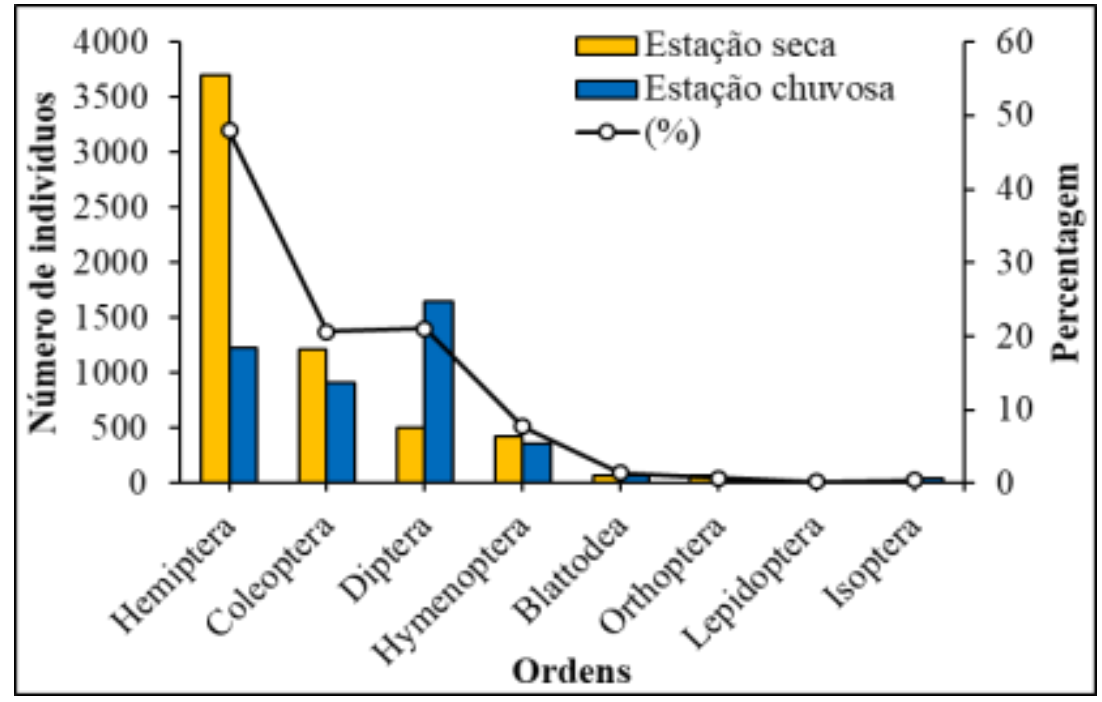

Fonte: Autores.

Do total de insetos coletados, 58,37\% foram amostrados na estação seca e 41,63\% na estação chuvosa. As ordens que apresentaram o maior número de insetos coletados na estação seca foram a ordem Hemiptera com 1.135 indivíduos em $E$. urophylla e 2.567 no híbrido, seguido pela ordem Coleoptera, 713 e 501 indivíduos, respectivamente e a ordem Diptera, com 325 e 181 indivíduos coletados respectivamente (Figura 3). As famílias de insetos mais abundantes neste mesmo período para E. urophylla foram Cicadellidae (33,5\%), Coccinellidae (15\%), Aphalaridae (6,3\%) e Formicidae (5\%). Para o híbrido, as famílias mais abundantes foram Cicadellidae (63,85\%), Coccinellidae (7,54\%), Formicidae (4\%) e Chrysomellidae (3,4\%). Essas famílias representaram $64,6 \%$ e $78,76 \%$ de toda a entomofauna coletada durante a estação seca em E. urophylla e no clone híbrido, respectivamente.

Figura 3. Principais ordens de insetos amostradas na estação seca (A) e chuvosa (B), em áreas comerciais de Eucalyptus urophylla e do híbrido E. grandis x E. urophylla, na Amazônia Oriental, Brasil.

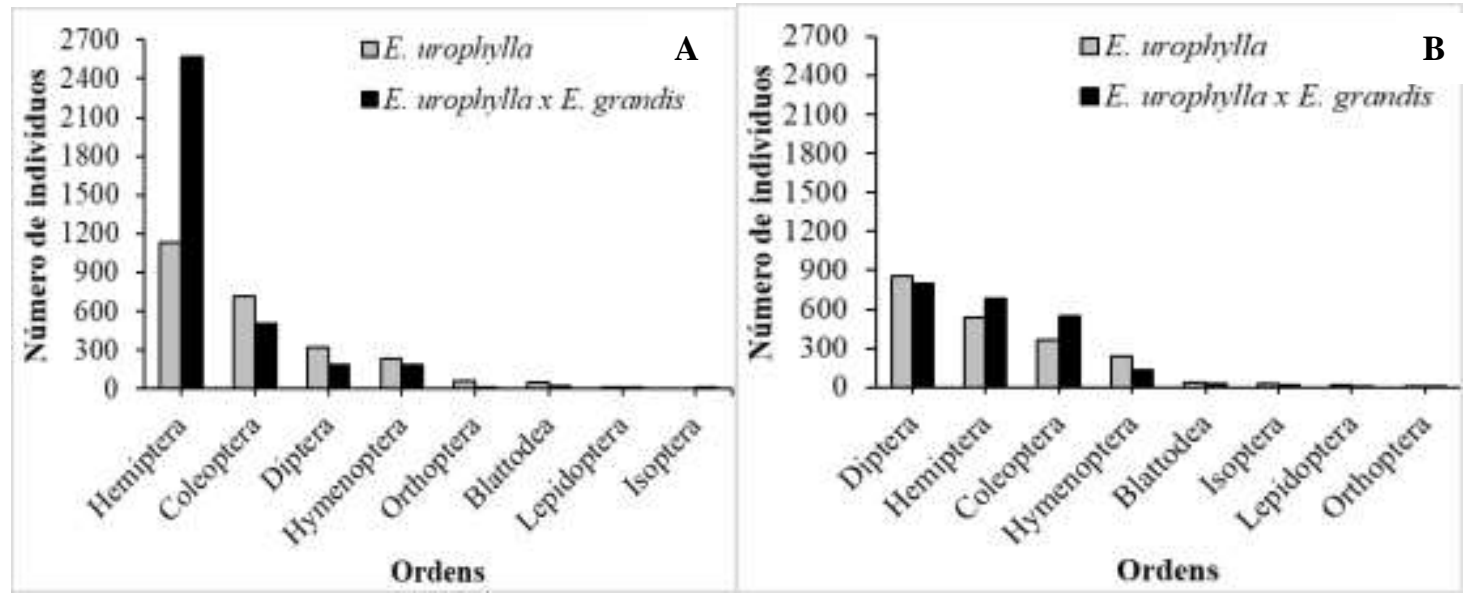

Fonte: Autores.

As ordens que apresentaram o maior número de insetos coletados na estação chuvosa foram Diptera com 857 e 798 indivíduos para E. urophylla e o híbrido, respectivamente; em seguida Hemiptera com 542 e 684 indivíduos coletados e 
Coleoptera com 365 e 544 indivíduos. Com destaque para as famílias Culicidae (21\%), Cicadellidae (13,7\%), Muscidae (12,3\%), Aphididae $(7,4 \%)$ e Formicidae $(4,9 \%)$ em E. urophylla. No híbrido, as principais famílias foram Cicadellidae (24,36\%), Culicidae (14,34\%), Muscidae (12,1\%), Chrysomelidae (10,6\%) e Formicidae (4,2\%). O somatório do percentual de insetos coletados nestas famílias corresponde a 59,3\% e 65,6\% de toda a entomofauna coletada durante a estação chuvosa em E. urophylla e no clone híbrido, respectivamente. As demais ordens, Blattodea, Isoptera, Lepidoptera e Orthoptera apresentaram o menor número de insetos coletados, nas duas estações climáticas.

De acordo com a análise estatística não foi observada diferença significativa entre as estações climáticas e entre os clones estudados, o que pode estar relacionado ao reduzido número de fatores analisados neste estudo, bem como ao número de insetos coletados.

Figura 4. Análise Fatorial do número de insetos amostrados em Eucalyptus urophylla e no híbrido E. grandis x E. urophylla, na Amazônia Oriental, Brasil.

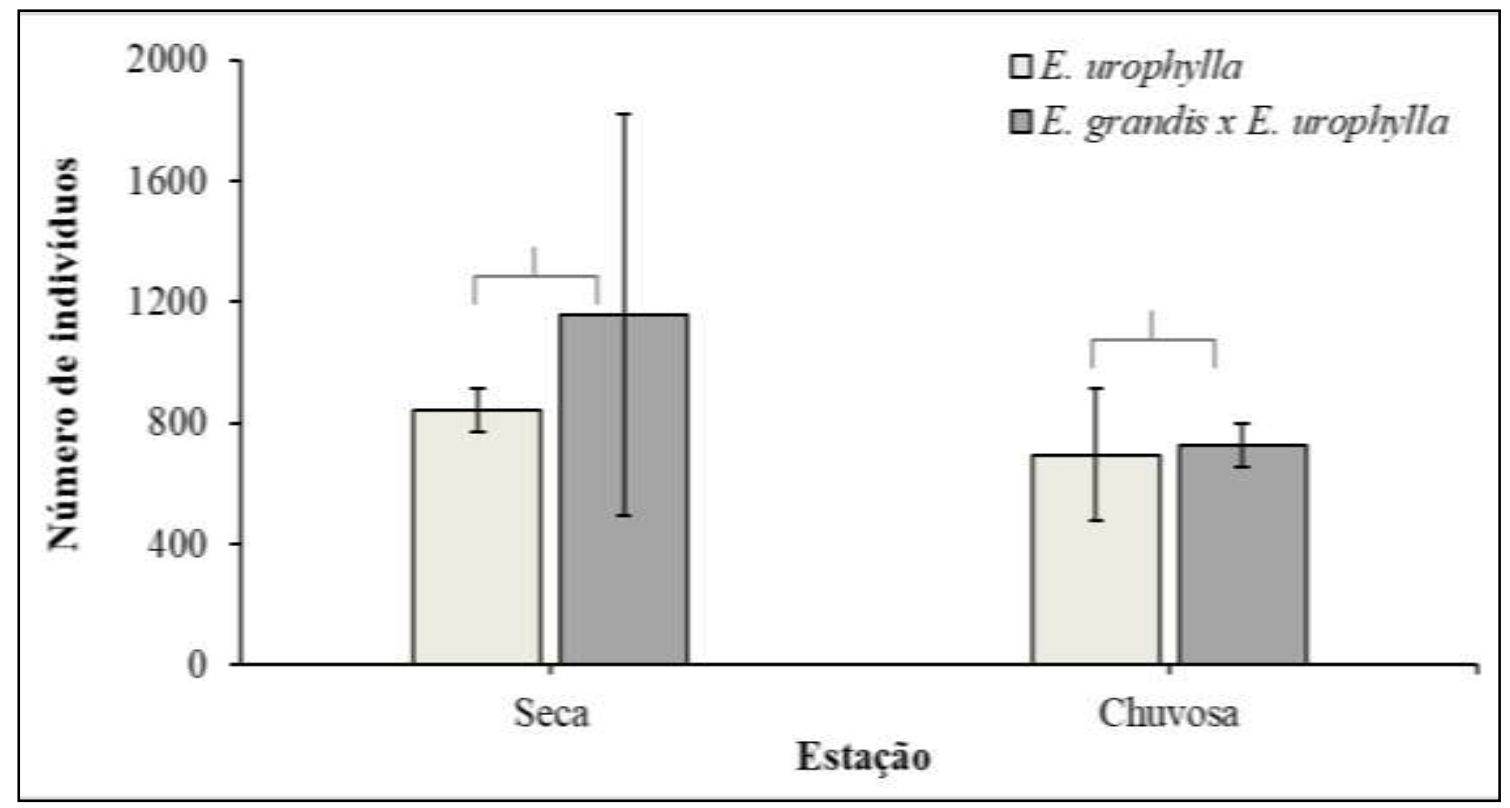

Letras maiúsculas e minúsculas iguais não diferem estatisticamente a 5\% de significância, de acordo com o teste de Análise Fatorial 2 x 2. Fonte: Autores.

\section{Ambientes}

O índice de diversidade de Shannon (H’) foi semelhante para os dois plantios avaliados (Tabela 1). 
Tabela 1. Índices faunísticos em relação a ordens de insetos coletados em plantios comerciais de E. urophylla e do híbrido E. urophylla x E. grandis, Amazônia Oriental, Brasil.

\begin{tabular}{ccc}
\hline Índices Faunísticos & E. urophylla & E. grandis x E. urophylla \\
\hline Índice de Shannon $\left(H^{\prime}\right)$ & 2,96 & 3,08 \\
\hline Índice de Equabilidade $\left(J^{\prime}\right)$ & 0,72 & 0,74 \\
\hline
\end{tabular}

Fonte: Autores.

\section{Diversidade}

A partir dos índices faunísticos para as famílias encontradas neste estudo (Tabela 2), pode-se constatar que as famílias com maiores índices pertencem às ordens Coleoptera, Diptera, Hemiptera e Hymenoptera para E. urophylla e o híbrido. As famílias Chrysomelidae, Coccinellidae e Scarabaeidae (Coleoptera) destacaram-se com índices significativos, ocorrendo em todas as coletas realizadas. As famílias apresentaram índices diferentes nos dois plantios. Chrysomelidae e Coccinellidae mostraram-se dominantes, muito abundantes, muito frequentes e constantes em E. urophylla e no híbrido, enquanto a família Scarabaeidae foi dominante, muito abundante, muito frequente e constante apenas no híbrido, indicando a preferência deste grupo para esta espécie de eucalipto. Na ordem Diptera, as famílias Ceratopogonidae, Culicidae e Muscidae apresentaram índices significativos, sendo a única que se manteve dominante, muito abundante, muito frequente e constante nos dois plantios estudados.

$\mathrm{Na}$ ordem Hemiptera, as famílias Aphididae, Aphalaridae e Cicadellidae mantiveram-se dominantes, muito abundantes, muito frequentes e constantes nos plantios de E. urophylla, e as famílias Aphidade e Membracidae apenas no híbrido. Sendo que a família Cicadellidae apresentou índices muito significativos no híbrido, mostrando-se superdominante, superabundante, super frequente e constante. A família Thaumastocoridae mostrou-se dominante, comum, frequente e constante para os dois plantios estudados. Pode-se confirmar a preferência das famílias Membracidae e Cicadellidae por plantios de E. grandis $x$ E. urophylla. A ordem Hymenoptera apresenta destaque para a família Formicidae como dominante, muito abundante, muito frequente e constante nos dois plantios de eucalipto avaliados.

Tabela 2. Índices faunísticos para ordens de insetos amostrados em Eucalyptus urophylla e no híbrido E. grandis x E. urophylla, na Amazônia Oriental, Brasil, quanto à dominância (D), abundância (A), frequência (F) e constância (C). D: não dominante (ND), dominante (D) e superdominante (SD). A: rara (r), dispersa (d), comum (c), abundante (a), muito abundante (ma) e superabundante (sa). F: pouco frequente (pf), frequente (f), muito frequente (mf), super frequente (sf). C: espécies constantes $(\mathrm{X})$, espécies acessórias $(\mathrm{Y})$ e espécies acidentais $(\mathrm{Z})$.

\begin{tabular}{|c|c|c|c|c|c|c|c|c|c|c|c|c|c|}
\hline \multirow[t]{2}{*}{ Ordem } & \multirow[t]{2}{*}{ Família } & \multicolumn{5}{|c|}{ E. urophylla } & \multicolumn{5}{|c|}{$\begin{array}{c}\text { E. grandis } \\
\mathbf{x} \\
\text { E. urophylla }\end{array}$} & \multirow{2}{*}{$\begin{array}{c}\text { Total } \\
\text { de } \\
\text { Indivíduos }\end{array}$} & \multirow[t]{2}{*}{$\%$} \\
\hline & & Total & D & $\mathbf{A}$ & $\mathbf{F}$ & $\mathbf{C}$ & Total & D & $\mathbf{A}$ & $\mathbf{F}$ & $\mathbf{C}$ & & \\
\hline Blattodea & Blatidae & 95 & $\mathrm{D}$ & $\mathrm{c}$ & $\mathrm{F}$ & W & 46 & $\mathrm{D}$ & $\mathrm{C}$ & $\mathrm{F}$ & W & 141 & 1,37 \\
\hline \multirow[t]{6}{*}{ Coleoptera } & Anobiidae & 0 & - & - & - & - & 3 & ND & $\mathrm{R}$ & $\mathrm{PF}$ & $\mathrm{Z}$ & 3 & 0,03 \\
\hline & Bostrichidae & 15 & $\mathrm{D}$ & $\mathrm{r}$ & $\mathrm{pf}$ & $\mathrm{W}$ & 6 & $\mathrm{D}$ & $\mathrm{R}$ & PF & W & 21 & 0,20 \\
\hline & Buprestidae & 19 & $\mathrm{D}$ & $\mathrm{r}$ & $\mathrm{pf}$ & $\mathrm{W}$ & 7 & $\mathrm{D}$ & $\mathrm{R}$ & PF & $\mathrm{Y}$ & 26 & 0,25 \\
\hline & Burchidae & 56 & $\mathrm{D}$ & $\mathrm{c}$ & $\mathrm{F}$ & $\mathrm{W}$ & 26 & $\mathrm{D}$ & $\mathrm{C}$ & $\mathrm{F}$ & $\mathrm{W}$ & 82 & 0,80 \\
\hline & Carabidae & 55 & $\mathrm{D}$ & $\mathrm{c}$ & $\mathrm{F}$ & $\mathrm{W}$ & 38 & $\mathrm{D}$ & $\mathrm{C}$ & $\mathrm{F}$ & $\mathrm{W}$ & 93 & 0,90 \\
\hline & Cerambicidae & 9 & $\mathrm{D}$ & $\mathrm{r}$ & $\mathrm{PF}$ & $\mathrm{Y}$ & 1 & ND & $\mathrm{R}$ & $\mathrm{PF}$ & $\mathrm{Z}$ & 10 & 0,10 \\
\hline
\end{tabular}




\begin{tabular}{|c|c|c|c|c|c|c|c|c|c|c|c|c|c|}
\hline & Chrysomelidae & 201 & $\mathrm{D}$ & $\mathrm{ma}$ & $\mathrm{MF}$ & $\mathrm{W}$ & 351 & $\mathrm{D}$ & $\mathrm{ma}$ & $\mathrm{MF}$ & $\mathrm{W}$ & 552 & 5,37 \\
\hline & Coccinellidae & 448 & $\mathrm{D}$ & $\mathrm{ma}$ & $\mathrm{MF}$ & $\mathrm{W}$ & 347 & $\mathrm{D}$ & $\mathrm{ma}$ & $\mathrm{MF}$ & $\mathrm{W}$ & 795 & 7,73 \\
\hline & Curculionidae & 77 & $\mathrm{D}$ & $\mathrm{c}$ & F & W & 61 & $\mathrm{D}$ & $\mathrm{C}$ & $\mathrm{F}$ & W & 138 & 1,34 \\
\hline & Elateridae & 20 & $\mathrm{D}$ & $\mathrm{r}$ & $\mathrm{PF}$ & W & 19 & $\mathrm{D}$ & $\mathrm{D}$ & $\mathrm{PF}$ & $\mathrm{W}$ & 39 & 0,38 \\
\hline & Hydrophilidae & 4 & ND & $r$ & $\mathrm{PF}$ & $\mathrm{Z}$ & 2 & ND & $\mathrm{R}$ & $\mathrm{PF}$ & $\mathrm{Z}$ & 6 & 0,06 \\
\hline & Lagridae & 10 & D & $\mathrm{r}$ & $\mathrm{PF}$ & $\mathrm{Y}$ & 1 & ND & $\mathrm{R}$ & $\mathrm{PF}$ & $\mathrm{Z}$ & 11 & 0,11 \\
\hline & Lampyridae & 4 & ND & $\mathrm{r}$ & $\mathrm{PF}$ & $\mathrm{Y}$ & 12 & $\mathrm{D}$ & $\mathrm{R}$ & $\mathrm{PF}$ & $\mathrm{W}$ & 16 & 0,16 \\
\hline & Lycidae & 2 & ND & $\mathrm{r}$ & $\mathrm{PF}$ & $\mathrm{Z}$ & 3 & ND & $\mathrm{R}$ & $\mathrm{PF}$ & $\mathrm{Y}$ & 5 & 0,05 \\
\hline & Meloidae & 11 & $\mathrm{D}$ & $\mathrm{r}$ & PF & W & 5 & ND & $\mathrm{R}$ & $\mathrm{PF}$ & $\mathrm{Y}$ & 16 & 0,16 \\
\hline & Nitidulidae & 5 & ND & $r$ & $\mathrm{PF}$ & $\mathrm{Y}$ & 6 & $\mathrm{D}$ & $\mathrm{R}$ & $\mathrm{PF}$ & $\mathrm{W}$ & 11 & 0,11 \\
\hline & Passalidae & 0 & - & - & - & - & 1 & ND & $\mathrm{R}$ & $\mathrm{PF}$ & $\mathrm{Z}$ & 1 & 0,01 \\
\hline & Scarabaeidae & 90 & $\mathrm{D}$ & $\mathrm{c}$ & $\mathrm{F}$ & W & 100 & $\mathrm{D}$ & $\mathrm{ma}$ & MF & $\mathrm{W}$ & 190 & 1,85 \\
\hline & Staphylinidae & 31 & $\mathrm{D}$ & d & $\mathrm{PF}$ & W & 29 & $\mathrm{D}$ & $\mathrm{C}$ & $\mathrm{F}$ & $\mathrm{W}$ & 60 & 0,58 \\
\hline & Tenebrionidae & 21 & $\mathrm{D}$ & $\mathrm{r}$ & $\mathrm{PF}$ & $\mathrm{W}$ & 27 & $\mathrm{D}$ & $\mathrm{C}$ & $\mathrm{F}$ & $\mathrm{W}$ & 48 & 0,47 \\
\hline Diptera & Azilidae & 0 & - & - & - & - & 2 & ND & $\mathrm{R}$ & $\mathrm{F}$ & $\mathrm{Y}$ & 2 & 0,02 \\
\hline & Caliphoridae & 1 & ND & $\mathrm{r}$ & $\mathrm{PF}$ & $\mathrm{W}$ & 3 & ND & $\mathrm{R}$ & $\mathrm{F}$ & $\mathrm{Y}$ & 4 & 0,04 \\
\hline & Ceratopogonidae & 125 & $\mathrm{D}$ & $\mathrm{ma}$ & MF & W & 86 & $\mathrm{D}$ & $\mathrm{ma}$ & MF & $\mathrm{W}$ & 211 & 2,05 \\
\hline & Culicidae & 440 & $\mathrm{D}$ & $\mathrm{ma}$ & MF & W & 330 & $\mathrm{D}$ & $\mathrm{ma}$ & MF & W & 770 & 7,49 \\
\hline & Dolichopodidae & 57 & $\mathrm{D}$ & $\mathrm{c}$ & $\mathrm{F}$ & W & 41 & D & $\mathrm{C}$ & $\mathrm{F}$ & $\mathrm{W}$ & 98 & 0,95 \\
\hline & Drosophilidae & 31 & $\mathrm{D}$ & $\mathrm{d}$ & PF & W & 34 & $\mathrm{D}$ & $\mathrm{C}$ & $\mathrm{F}$ & $\mathrm{W}$ & 65 & 0,63 \\
\hline & Lonchaeidae & 41 & $\mathrm{D}$ & $\mathrm{c}$ & $\mathrm{F}$ & W & 13 & $\mathrm{D}$ & $\mathrm{R}$ & $\mathrm{PF}$ & $\mathrm{Y}$ & 54 & 0,53 \\
\hline & Muscidae & 313 & $\mathrm{D}$ & $\mathrm{ma}$ & MF & W & 354 & $\mathrm{D}$ & $\mathrm{ma}$ & MF & $\mathrm{W}$ & 667 & 6,49 \\
\hline & Stratiomyidae & 0 & - & - & - & - & 1 & ND & $\mathrm{R}$ & $\mathrm{PF}$ & $\mathrm{Z}$ & 1 & 0,01 \\
\hline & Tabanidae & 1 & ND & $\mathrm{R}$ & $\mathrm{PF}$ & $\mathrm{Z}$ & 0 & - & - & - & - & 1 & 0,01 \\
\hline & Tephritidae & 77 & $\mathrm{D}$ & $\mathrm{C}$ & $\mathrm{F}$ & W & 14 & $\mathrm{D}$ & $\mathrm{R}$ & $\mathrm{PF}$ & $\mathrm{W}$ & 91 & 0,89 \\
\hline & Tipulidae & 12 & $\mathrm{D}$ & $\mathrm{R}$ & PF & W & 5 & ND & $\mathrm{R}$ & $\mathrm{PF}$ & $\mathrm{Y}$ & 17 & 0,17 \\
\hline & Trichogrammatidae & 20 & $\mathrm{D}$ & $\mathrm{R}$ & $\mathrm{PF}$ & W & 66 & $\mathrm{D}$ & $\mathrm{C}$ & $\mathrm{F}$ & $\mathrm{W}$ & 86 & 0,84 \\
\hline & Ulidiidae & 64 & $\mathrm{D}$ & $\mathrm{C}$ & $\mathrm{F}$ & $\mathrm{W}$ & 30 & $\mathrm{D}$ & $\mathrm{C}$ & $\mathrm{F}$ & $\mathrm{W}$ & 94 & 0,91 \\
\hline Hemiptera & Aphididae & 177 & $\mathrm{D}$ & $\mathrm{ma}$ & MF & $\mathrm{W}$ & 96 & $\mathrm{D}$ & $\mathrm{ma}$ & MF & $\mathrm{W}$ & 273 & 2,66 \\
\hline & Aphalaridae & 168 & $\mathrm{D}$ & $\mathrm{ma}$ & MF & W & 34 & $\mathrm{D}$ & $\mathrm{C}$ & $\mathrm{F}$ & $\mathrm{W}$ & 202 & 1,96 \\
\hline & Berytidae & 1 & ND & $\mathrm{R}$ & $\mathrm{PF}$ & $\mathrm{Z}$ & 1 & $\mathrm{ND}$ & $\mathrm{R}$ & $\mathrm{PF}$ & $\mathrm{Z}$ & 2 & 0,02 \\
\hline & Cercopidae & 23 & $\mathrm{D}$ & $\mathrm{D}$ & $\mathrm{PF}$ & W & 59 & $\mathrm{D}$ & $\mathrm{C}$ & $\mathrm{F}$ & $\mathrm{W}$ & 82 & 0,80 \\
\hline & Cicadellidae & 1132 & $\mathrm{D}$ & $\mathrm{ma}$ & MF & W & 2753 & SD & $\mathrm{sa}$ & SF & $\mathrm{W}$ & 3885 & 37,79 \\
\hline & Cicadidae & 1 & ND & $\mathrm{R}$ & $\mathrm{PF}$ & $\mathrm{Z}$ & 1 & ND & $\mathrm{R}$ & $\mathrm{PF}$ & $\mathrm{Z}$ & 2 & 0,02 \\
\hline & Delphacidae & 4 & ND & $\mathrm{R}$ & PF & $\mathrm{Z}$ & 2 & ND & $\mathrm{R}$ & $\mathrm{PF}$ & $\mathrm{Z}$ & 6 & 0,06 \\
\hline & Dictyopharidae & 0 & - & - & - & - & 2 & ND & $\mathrm{R}$ & $\mathrm{PF}$ & $\mathrm{Z}$ & 2 & 0,02 \\
\hline & Flatidae & 0 & - & - & - & - & 1 & ND & $\mathrm{R}$ & PF & $\mathrm{Z}$ & 1 & 0,01 \\
\hline & Gerridae & 0 & - & - & - & - & 2 & ND & $\mathrm{R}$ & $\mathrm{PF}$ & $Y$ & 2 & 0,02 \\
\hline & Largidae & 12 & $\mathrm{D}$ & $\mathrm{R}$ & PF & W & 26 & $\mathrm{D}$ & $\mathrm{C}$ & $\mathrm{F}$ & W & 38 & 0,37 \\
\hline & Lygaeidae & 4 & ND & $\mathrm{R}$ & $\mathrm{PF}$ & W & 1 & ND & $\mathrm{R}$ & $\mathrm{PF}$ & $\mathrm{Z}$ & 5 & 0,05 \\
\hline & Membracidae & 66 & D & $\mathrm{C}$ & $\mathrm{F}$ & W & 111 & D & $\mathrm{ma}$ & MF & W & 177 & 1,72 \\
\hline & Miridae & 0 & - & - & - & - & 5 & ND & $\mathrm{R}$ & PF & Y & 5 & 0,05 \\
\hline & Pentatomidae & 1 & ND & $\mathrm{R}$ & PF & $\mathrm{Z}$ & 0 & - & - & - & - & 1 & 0,01 \\
\hline & Psyllidae & 3 & ND & $\mathrm{R}$ & $\mathrm{PF}$ & $\mathrm{Z}$ & 60 & $\mathrm{D}$ & $\mathrm{C}$ & $\mathrm{F}$ & W & 63 & 0,61 \\
\hline & Pyrrhocoridae & 7 & $\mathrm{D}$ & $\mathrm{R}$ & $\mathrm{PF}$ & $\mathrm{W}$ & 13 & $\mathrm{D}$ & $\mathrm{R}$ & $\mathrm{PF}$ & W & 20 & 0,19 \\
\hline
\end{tabular}




\begin{tabular}{|c|c|c|c|c|c|c|c|c|c|c|c|c|c|}
\hline & Scuteleridae & 7 & $\mathrm{D}$ & $\mathrm{R}$ & $\mathrm{PF}$ & W & 29 & $\mathrm{D}$ & $\mathrm{C}$ & $\mathrm{F}$ & $\mathrm{W}$ & 36 & 0,35 \\
\hline & Thaumastocoridae & 63 & $\mathrm{D}$ & $\mathrm{C}$ & $\mathrm{F}$ & W & 52 & $\mathrm{D}$ & $\mathrm{C}$ & $\mathrm{F}$ & $\mathrm{W}$ & 115 & 1,12 \\
\hline & Tingidae & 8 & $\mathrm{D}$ & $\mathrm{R}$ & $\mathrm{PF}$ & $\mathrm{Y}$ & 3 & ND & $\mathrm{R}$ & $\mathrm{PF}$ & $\mathrm{W}$ & 11 & 0,11 \\
\hline \multirow[t]{7}{*}{ Hymenoptera } & Chalcididae & 11 & $\mathrm{D}$ & $\mathrm{R}$ & $\mathrm{PF}$ & W & 3 & ND & $\mathrm{R}$ & $\mathrm{PF}$ & $\mathrm{Y}$ & 14 & 0,14 \\
\hline & Crabronidae & 124 & $\mathrm{D}$ & A & MF & W & 16 & $\mathrm{D}$ & $\mathrm{R}$ & $\mathrm{PF}$ & $\mathrm{W}$ & 140 & 1,36 \\
\hline & Evaniidae & 5 & ND & $\mathrm{R}$ & $\mathrm{PF}$ & $\mathrm{Y}$ & 1 & ND & $\mathrm{R}$ & $\mathrm{PF}$ & $\mathrm{Z}$ & 6 & 0,06 \\
\hline & Formicidae & 229 & $\mathrm{D}$ & $\mathrm{ma}$ & MF & W & 231 & $\mathrm{D}$ & $\mathrm{ma}$ & MF & $\mathrm{W}$ & 460 & 4,47 \\
\hline & Ichneumonidae & 12 & $\mathrm{D}$ & $\mathrm{R}$ & $\mathrm{PF}$ & W & 2 & ND & $\mathrm{R}$ & $\mathrm{PF}$ & $\mathrm{Y}$ & 14 & 0,14 \\
\hline & Sphecidae & 26 & $\mathrm{D}$ & $\mathrm{D}$ & $\mathrm{PF}$ & $\mathrm{W}$ & 24 & $\mathrm{D}$ & $\mathrm{D}$ & $\mathrm{PF}$ & $\mathrm{W}$ & 50 & 0,49 \\
\hline & Vespidae & 68 & $\mathrm{D}$ & $\mathrm{C}$ & $\mathrm{F}$ & W & 41 & $\mathrm{D}$ & $\mathrm{C}$ & $\mathrm{F}$ & $\mathrm{W}$ & 109 & 1,06 \\
\hline Isoptera & Rhinotermitidae & 26 & $\mathrm{D}$ & $\mathrm{D}$ & $\mathrm{PF}$ & W & 15 & $\mathrm{D}$ & $\mathrm{R}$ & $\mathrm{PF}$ & $\mathrm{W}$ & 41 & 0,40 \\
\hline \multirow[t]{2}{*}{ Lepidoptera } & Hesperiidae & 1 & ND & $\mathrm{R}$ & $\mathrm{PF}$ & $\mathrm{Z}$ & 1 & ND & $\mathrm{R}$ & $\mathrm{PF}$ & $\mathrm{Z}$ & 2 & 0,02 \\
\hline & Pieridae & 2 & ND & $\mathrm{R}$ & $\mathrm{PF}$ & $\mathrm{Y}$ & 2 & ND & $\mathrm{R}$ & $\mathrm{PF}$ & $\mathrm{Y}$ & 4 & 0,04 \\
\hline \multirow[t]{4}{*}{ Orthoptera } & Acrididae & 1 & ND & $\mathrm{R}$ & $\mathrm{PF}$ & $\mathrm{Z}$ & 1 & ND & $\mathrm{R}$ & $\mathrm{PF}$ & $\mathrm{Z}$ & 2 & 0,02 \\
\hline & Gryllidae & 2 & ND & $\mathrm{R}$ & $\mathrm{PF}$ & $\mathrm{Y}$ & 9 & $\mathrm{D}$ & $\mathrm{R}$ & $\mathrm{PF}$ & $\mathrm{Y}$ & 11 & 0,11 \\
\hline & Tettigoniidae & 71 & $\mathrm{D}$ & $\mathrm{C}$ & $\mathrm{F}$ & W & 3 & ND & $\mathrm{R}$ & $\mathrm{PF}$ & $\mathrm{W}$ & 74 & 0,72 \\
\hline & Total & 4610 & & & & & 5670 & & & & & 10280 & 100 \\
\hline
\end{tabular}

Fonte: Autores.

\section{Discussão}

\section{Sazonalidade}

Foram coletados 10.280 indivíduos, em oito ordens e 68 famílias, com destaque para as ordens Hemiptera, Diptera e Coleoptera. As ordens Coleoptera, Diptera, Hemiptera e Hymenoptera destacam-se pela riqueza de espécies, justificando o elevado número de insetos coletados. Mendel e Protasov (2019) relatam que a ordem Hemiptera é o principal grupo presente em plantios de Eucalyptus spp., seguido pela ordem Coleoptera.

Diferenças entre armadilhas influenciam na quantidade de indivíduos amostrados em cada ordem. Armadilhas adesivas amarelas podem apresentar as ordens Diptera e Hymenoptera, com mais de $90 \%$ do total da amostra. O amarelo é considerado mais atrativo para muitos grupos de insetos, principalmente Diptera. Os resultados do presente estudo foram semelhantes aos de Gonzaga et al. (2021), que encontrou as ordens Coleoptera, Hemiptera, Hymenoptera e Diptera com os maiores números de insetos coletados em plantação de eucalipto em Rio Largo, AL. Garlet et al. (2016), encontraram as ordens Coleoptera, Lepidoptera e Hemiptera como as mais abundantes em plantios de Eucalyptus spp. em São Francisco de Assis, RS, utilizando armadilhas luminosas Luiz de Queiroz instaladas a 1,5 m do solo. Nunes et al. (2017) avaliando a entomofauna em Patrocínio, MG, por meio de armadilhas coloridas, encontraram as ordens Diptera e Coleoptera, como os grupos mais abundantes.

A estação seca apresentou maior número de indivíduos, o que evidencia a interferência da sazonalidade durante o período de coleta. As condições secas associadas às mudanças climáticas influenciam no aumento da atividade populacional de espécies de insetos florestais. Os períodos de elevadas temperaturas acarretam altas densidades populacionais, somado a presença de hospedeiros e a capacidade de dispersão das espécies.

Rodrigues (2004) considera a faixa entre 15 e $38^{\circ} \mathrm{C}$ ótima para o desenvolvimento de espécies de insetos, portanto, o aumento da temperatura e da umidade relativa do ar possivelmente influenciou no aumento do número de insetos coletados no período seco, reduzindo a taxa de evaporação e mantendo condições favoráveis à ocorrência dos artrópodes. 
A ordem Hemiptera apresentou o maior número de insetos capturados no período seco. Ambas possuem famílias importantes de pragas para o cultivo de Eucalyptus spp., sendo a primeira com insetos heliotérmicos e que aproveitam ao máximo os raios solares para aumentar a temperatura do seu corpo (Costa et al, 2014), o que pode justificar a sua maior ocorrência no período seco. A ordem Hemiptera incluí as principais espécies fitófagas que podem inocular substâncias tóxicas nas plantas em plantios de eucalipto, causando danos diretos ou indiretos ao plantio. Espécies de insetos-praga exóticas foram registradas nos dois plantios de Eucalyptus spp.: Glycaspis brimblecombei e Blastopsylla occidentalis (Aphalaridae) e Thaumastocoris peregrinus (Thaumastocoridae). Os monocultivos florestais com alta densidade de plantas e que são cultivados por longos períodos oferecem condições propícias para o estabelecimento e dispersão de espécies-praga, principalmente em cultivos com espécies de Eucalyptus (Barbosa et al., 2021).

A ordem Coleoptera foi a segunda a exibir o maior número de insetos coletados no período seco. Segundo Silveira Neto et al. (1976), o aumento da temperatura favorece maior movimentação e dispersão dos besouros, mostrando que são insetos adaptados a altas temperaturas. Coleópteros são considerados bioindicadores em estudos entomológicos e variam seus picos populacionais, conforme as condições ambientais, como variações na temperatura e precipitação pluvial que influenciam na emergência. Coleópteros são sensíveis a mudanças ambientais, pois estas afetam diretamente a riqueza, distribuição, abundância e até a estrutura de suas guildas. Correia (2018) encontrou o maior número de insetos coletados pertencentes à ordem Coleoptera no período chuvoso, diferindo dos resultados encontrados no presente estudo.

Em termos de estação chuvosa, Costa et al. (2014) destacam que a chuva pode ter ação negativa, principalmente sobre insetos pequenos e, dependendo da intensidade, pode atuar também sobre os insetos maiores. No entanto, Correia (2018) destacam que a estação chuvosa é considerada fator preponderante para os níveis populacionais de insetos em ecossistemas florestais e a precipitação é um dos fatores climáticos mais importantes envolvendo a suscetibilidade das árvores hospedeiras a insetos florestais, comprometendo as defesas e o vigor das árvores.

Insetos das ordens Diptera tiveram o maior número de indivíduos coletados no período chuvoso. Os dípteros possuem hábitos variados, larvas e adultos e podem sobreviver em ambientes adversos, sendo tolerantes a impactos ambientais (Gallo et al., 2013). Resultados semelhantes foram encontrados por Nunes et al. (2017), onde a ordem Diptera foi a mais abundante. A grande quantidade de insetos coletados pode ser justificada pela grande diversidade que esse grupo de insetos apresenta, tanto ecologicamente quanto em termos de riqueza de espécies. Alguns estudos relataram que a ordem Diptera é o grupo mais abundante e, determinam as flutuações locais (Bordin \& Sartor, 2016).

As demais ordens, Blattodea, Isoptera, Lepidoptera e Orthoptera apresentaram menor número de insetos, nos dois períodos. Este resultado pode estar relacionado à metodologia de coleta utilizada (Nunes et al., 2017).

\section{Ambientes}

O Índice de Shannon indicou que ambos os ambientes são propícios para a ocorrência das ordens e famílias de insetos encontrados neste estudo, favorecendo a ocorrência natural de insetos, sejam estes inimigos naturais ou insetos-praga. Resultados semelhantes ao do presente estudo foram encontrados nos estudos de Garlet et al. (2016) e Gonzaga et al. (2021). O aumento no valor do índice de Shannon-Weaner ( $\left.H^{\prime}\right)$ pode ser decorrente do aumento na riqueza de espécies, na uniformidade dos valores através de todas as amostras ou de ambas as características (Magurran, 2011).

Os dois plantios exibiram valor do índice de Equabilidade de Pielou (J') semelhantes, com uniformidade entre si, pois são ambientes de monocultivos florestais. A equabilidade indica uma uniformidade na abundância de famílias, podendo ser amostrada uma comunidade mais homogênea, possivelmente relacionada à ausência de predadores ou indivíduos competidores. O estudo da diversidade de insetos em plantios de eucalipto bem como sua dinâmica populacional permite determinar as principais espécies de ocorrência nestes cultivos e os seus principais picos populacionais. Estes resultados podem 
ser correlacionados com variáveis bióticas e abióticas que influenciam na escolha da melhor tomada de decisão frente ao emprego de diferentes métodos de controle (Lima, 1996).

\section{Diversidade}

Gonzaga et al. (2021) encontrou a família Chrysomelliae como dominante, muito abundante, muito frequente. Garlet et al. (2016), encontraram a ordem Coleoptera e as famílias Chrysomelidae e Scarabaeidae em plantios de E. grandis x E. urophylla, resultado semelhante a este estudo. Esta ordem pode sofrer variações pelo tipo de cobertura vegetal, estrutura da vegetação, tipo de solo e composição florística, variando em abundância de indivíduos, riqueza de espécies e diversidade.

Os coleópteros são importantes para a cultura do eucalipto, pois incluem várias espécies consideradas pragas da cultura, com destaque para os besouros desfolhadores pertencentes às famílias Chrysomelidae, Coccinellidae, Curculionidae e Scarabaeidae (Lunz \& Azevedo, 2016). Coccinelídeos também desempenham o papel como excelentes predadores de insetos fitófagos e outros insetos de pequeno porte (Honek et al., 2017). A família Scarabaeidae apresenta grande diversidade ecológica e alimentar, devido a seu hábito, esses insetos estão presentes na maioria dos ambientes.

As famílias Ceratopogonidae, Culicidae e Muscidae foram as mais abundantes, estas famílias, encontradas neste estudo são consideradas como agentes polinizadores, corroborado por Gullan e Cranston (2012). Insetos da ordem Diptera, possuem alta capacidade de colonização, principalmente no estádio larval (Gullan \& Cranston, 2012). Possuem insetos com alto potencial para a utilização como indicadores ambientais, pois exercem importantes funções como decompositores, polinizadores e controladores biológicos, fato que reforça sua importância (Brown, 1997). Os dípteros adaptados à polinização são conhecidos como polinizadores irregulares por não apresentar uma constância de visitação e podem ser atraídos pela cor amarela, pousando com frequência em flores desta coloração (Nunes et al., 2017).

A ordem Hemiptera compreende a maioria dos insetos fitófagos, considerada pragas agrícolas e florestais, como em plantios de eucalipto (Gullan \& Cranston, 2012; Costa et al., 2014). As famílias Aphididae, Aphalaridae e Cicadellidae apresentaram maior diversidade faunística para os insetos coletados em E. urophylla, e Thaumastocoridae apresentou ocorrência nos dois clones estudados, mantendo-se constante em todas as coletas realizadas. Em estudo realizado por Gonzaga et al. (2021), as famílias Membracidae e Cicadidae demonstram elevados índices de dominância, abundância, frequência e constância nos clones de E. urograndis avaliados, se assemelhando ao resultado encontrado no presente estudo.

A família Aphalaridae compreende as espécies-praga G. brimblecombei e B. occidentalis, que são minúsculos sugadores de seiva que se assemelham a pequenas cigarras. São capazes de causar danos diretos, senescência e a queda prematura das folhas, e danos indiretos, secretando uma substância açucarada que favorece o crescimento de fungos e que cobrem as folhas do hospedeiro, dificultando a fotossíntese dos indivíduos de eucalipto atacados (Barbosa et al., 2021). A família Thaumastocoridae compreende o percevejo bronzeado, T. peregrinus, espécie-praga de relevância econômica que pode causar seca da gema apical, queda das folhas e redução da capacidade fotossintética da árvore de espécies de eucalipto (Lunz \& Azevedo, 2016). Neste estudo, foram encontrados estas três espécies-praga, T. peregrinus (Saliba et al., 2019a), G. brimblecombei e B. occidentalis nos plantios de E. urophylla e E.grandis x E. urophylla (Saliba et al, 2019b).

Nunes et al. (2017) e Correia, (2018), encontraram a família Formicidae com maior abundância de indivíduos. A família está entre os invertebrados mais abundantes em ecossistemas terrestres, devido sua alta capacidade de sobrevivência. Constituem um grande grupo de importância econômica, com destaque às saúvas, que podem causar danos diretos e indiretos em eucalipto durante todo o ciclo florestal (Barbosa et al., 2021). A família Formicidae tme 334 gêneros, com 119 na região neotropical (Bolton, 2018). As formigas cortadeiras (Atta e Acromyrmex) compõem as pragas no Brasil, responsáveis por desfolhas intensas em eucalipto (Costa et al., 2014). Indivíduos de E. urophyla podem perder 16\% e 42\% do volume de madeira, aos 4,6 anos de idade, ao sofrerem desfolhas de $75 \%$ e $100 \%$, respectivamente. 


\section{Considerações Finais}

As ordens mais abundantes em plantios de Eucalyptus spp. na Amazônia Oriental foram Hemiptera, Coleoptera e Diptera. A ordem Hemíptera apresentou maior abundância de insetos na estação seca, e a ordem Diptera foi mais abundante na estação chuvosa. A sazonalidade influencia no número de insetos. Os resultados indicam que a estação seca é a mais indicada para a realização de estudos de levantamentos populacionais de insetos em plantios de Eucalyptus spp. na Amazônia Oriental.

Foi registrada a ocorrência das espécies-praga Blastopsylla occidentalis, Glycaspis brimblecombei, pertencentes à família Aphalaridae, e Thaumastocoris peregrinus, pertencente à família Thaumastocoridae, nos clones de E. urophylla e E. grandis $\mathrm{x}$ E. urophylla.

As famílias Chrysomelidae e Coccinellidae (Coleoptera); Ceratopogonidae, Culicidae e Muscidae (Diptera); Aphididae, Aphalaridae e Cicadellidae (Hemiptera) e a família Formicidae (Hymenoptera) apresentaram maior ocorrência em plantios de E. grandis x E. urophylla As famílias Chrysomelidae, Coccinellidae e Scarabaeidae (Coleoptera); Ceratopogonidae, Culicidae e Muscidae (Diptera); Aphididae, Cicadellidae e Membracidae (Hemiptera) e a família Formicidae (Hymenoptera) apresentaram maior ocorrência em plantios de E. grandis x E. urophylla.

Futuros estudos podem considerar o refinamento de análises nas variações populacionais sazonais das diferentes famílias e ordens da entomofauna aérea de Eucalyptus spp. As espécies-praga B. occidentalis, G. brimblecombei e T. peregrinus, podem ser monitoradas em mais detalhe, pois têm potencial de causar prejuízos substanciais em plantações de Eucalyptus spp. no estado do Pará.

\section{Referências}

Barbosa, L. R., Queiroz, D. L., Nickele, M. A., Queiroz, E. C., Filho, W. R., Iede, E. T., \& Penteado, S. R. C. (2021). Pragas de Eucaliptos. In: Oliveira, E. B., Pinto Junior, J. E. (eds.). Editores Técnicos. O eucalipto e a Embrapa: quatro décadas e pesquisa e desenvolvimento. Embrapa, 751 - 780.

Barbosa, L. R., Santos, F., Machado, B. O., Wilcken, C. F., Soliman, E. P., \& Zaché, B. (2012). Percevejo bronzeado do eucalipto: reconhecimento, danos e direcionamentos para o controle. Colombo: Embrapa Florestas, Documentos 239, 27p.

Bolton, B. (2018). Taxonomic History. < https://www.antweb.org/ description.do?name=formicidae\&rank=family\&project=allantwebants >

Bordin, D., \& Sartor, V. (2016). Diversidade e Abundância da entomofauna em 3 estações do ano no campus da Universidade do Contestado - UnC, Distrito de Marcílio Dias, Santa Catarina. Revista Saúde e Meio Ambiente, 5(1), 89-104.

Brown K. S. (1997). Diversity, disturbance, and sustainable use of Neotropical forests: insects as indicators for conservation monitoring. Journal of Insect Conservation, 1, 25-42.

Burckhardt, D., \& Queiroz, D. L. (2012). Checklist and comments on the jumping plant-lice (Hemiptera: Psylloidea) from Brazil. Zootaxa, 3571(1), 26-48.

Burckhardt, D., \& Ouvrard, D. (2012). A revised classification of the jumping plant-lice (Hemiptera: Psylloidea). Zootaxa, 3509(1), 1-34.

Carpintero, D. L., \& Dellapé, P. M. (2006). A new species of Thaumastocoris Kirkaldy from Argentina (Heteroptera: Thaumastocoridae: Thaumastocorinae). Zootaxa, 1228, 61-68.

Correia, R. G. (2018). Entomofauna Edáfica e armazenamento de Liteira em cultivos de Swietenia Macrophylla (King) na Amazônia Oriental. (Tese de Doutorado em Ciências Florestais). Universidade Federal Rural da Amazônia, 80p.

Costa, E. C., D’Avila, M., \& Cantarelli, E. B. (2014). Entomologia florestal. (3a ed.), UFSM, 256 p.

Dajoz, R. (1983). Ecologia geral. Vozes.

EMBRAPA - Empresa Brasileira De Pesquisa Agropecuaria. (2013). Sistema Brasileiro de Classificação de Solos. (3a ed.) Embrapa Solos, 306p.

Fazolin, M. (1991). Análise faunística de insetos coletados em seringueira no Acre. (Tese de Doutorado em Engenharia Florestal). Escola Superior de Agricultura Luiz de Queiroz, Universidade de São Paulo, 236p.

Foelkel, C. E. B. (2005). Eucalipto no Brasil, história de pioneirismo. Visão Agrícola, 4, 66-69.

FAPESPA - Fundação Amazônia De Amparo A Estudos E Pesquisas (2016). Estatísticas Municipais Paraenses: Dom Eliseu. Belém: Diretoria de Estatística e de Tecnologia e Gestão da Informação.

Gallo, D., Nakano, O., Neto, S. S., Carvalho, L. P. R., Baptista, C. G., Filho, B. E., Parra, P. R. J., Zucchi, A. R., Alves, B. S., Vendramim, D. J., Marchini, C. L., Lopes, S. R. J., \& Omoto, C. (2002). Entomologia Agrícola. FEALG. 
Garlet, J., Costa, E. C., \& Boscardin, J. (2016). Levantamento da entomofauna em plantios de Eucalyptus spp. por meio de armadilha luminosa em São Francisco de Assis - RS. Ciência Florestal, 26(2), 365-374.

Gómez, M. N. G., (2000). Metodologia da pesquisa no campo da Ciência da Informação. Revista de Ciência da Informação, 1(6), Artigo 3.

Gonzaga, E. P., Breda, M. O., Xavier, M. E. V., Santos, J. M., Santos, T. F., \& Santos, D. S. (2021). Diversidade inicial da entomofauna em povoamento florestal de eucalipto, no município de Rio Largo, Alagoas. Diversitas Journal, 6(3), 2931-2945.

Gullan, P. J., \& Cranston, P.S. (2012). Os insetos: um resumo de entomologia. (4a ed.), Roca, 480p.

Honek, A., Dixon, A. F. G., Soares, A. O., Skuhrovec, J., \& Martinkova, Z. (2017). Spatial and temporal changes in the abundance and compostion of ladybird (Coleoptera: Coccinellidae) communities. Current Opinion. Insect Science, 20, 61-67.

IBA - Indústria Brasileira de Árvores. (2020). Relatório Anual 2020. 66p.

IBGE - Instituto Brasileiro de Geografia e Estatística. (2012). Manual Técnico da Vegetação Brasileira. 271 p.

Lima, W.P. (1996). Impacto Ambiental do Eucalipto. (2a ed.) EDUSP.

Lunz, A. M., \& Azevedo, R. (2016). Eucalipto. In: Silva, N. M., Adaime, R., Zucchi, R. A. (eds.). Pragas agrícolas e florestais na Amazônia. Brasília: Embrapa, p.461 - 471 .

Lunz, A. M., Mourão Júnior, M., Monteiro, O. M., \& Souza, H. S. (2011). Entomofauna associada a reflorestamentos experimentais no município de Pau d'Arco, Pará. Ciência Rural, 41(12), 2101-2107.

Machado, C. A., \& Maia, K. (2017). Impactos ambientais da silvicultura em Dom Eliseu (PA). Revista Tocantinense de Geografia, 6(9), 157-173.

Magurran, A. E. (2011). Medindo a Diversidade Biológica. UFPR, 262p.

Mendel, Z., \& Protasov, A. (2019). The entomofauna on Eucalyptus in Israel: A review. European Journal of Entomology, 116, 450-460.

Nunes, M. S., Osório, M. F., Almeida, E. F., \& Oliveira, F. R. (2017). Avaliação de Entomofauna com Armadilhas Coloridas em Reserva Ecológica no Município de Patrocínio - MG. Revista Educação, Saúde e Meio Ambiente, 2, 158 - 175.

Pielou, E. C. (1966). Species diversity and pattern diversity in the study of ecological succession. Journal Theory Biology, 10, $370-383$.

R Core Team (2021). R: A language and environment for statistical computing. Vienna: R Foundation for Statistical Computing. https://www.R-project.org/.

Rodrigues, W. C. (2004). Fatores que influenciam o desenvolvimento dos insetos. Info Insetos, 4, 1-4.

Saliba, I. L., Lunz, A. M., Batista, T. F., \& Schwartz, G. (2019a). First record of Thaumastocoris peregrinus (Hemiptera, Thaumastocoridae) in Pará state, Brazil. Acta Amazônica, 49, 179-182.

Saliba, I. L., Lunz, A. M., Batista, T. F., Schwartz, G., \& Queiroz, D. L. (2019b). First record of Glycaspis brimblecombei (Moore, 1964) and Blastopsylla occidentalis (Taylor, 1985) (Hemiptera, Aphalaridae) in Eucalyptus plantations in State of Pará, Brazil. Entomological Communications, 1, 1-3.

Silveira Neto, S., Nakano, O., \& Vila Nova, N. A. (1976). Manual de ecologia dos insetos. Ceres, 419p.

SIPAM - Sistema de Proteção da Amazônia (2009). Zoneamento climático do Estado do Pará. Belém: Centro Técnico e Operacional de Belém - Pará, 30p.

Wilcken, C. F., Soliman, E. P., Nogueira de Sá, L. A., Barbosa, L., Dias, T. K. R., Ferreira Filho, P. J., \& Oliveira, R. J. R. (2010). Bronze bug Thaumastocoris peregrinus Carpintero \& Dellapé (Hemiptera: Thaumastocoridae) on Eucalyptus in Brazil and its distribution. Journal Research of Plant Protecion, 50(2), 184-188. 